Originalien

Nervenarzt 2021 · 92:349-358

https://doi.org/10.1007/s00115-020-01046-0

Angenommen: 10. Dezember 2020

Online publiziert: 5 . Januar 2021

๑) Der/die Autor(en) 2021

Burkhard Domurath ${ }^{1}$ Peter Flachenecker ${ }^{2}$. Thomas Henze ${ }^{3}$.

Wolfgang Feneberg ${ }^{4}$. Anna Brandt ${ }^{5}$. Ines Kurze ${ }^{6}$. Ruth Kirschner-Hermanns ${ }^{7}$. Albert Kaufmann $^{8} \cdot$ Jörn Bremer ${ }^{9} \cdot$ Manuela Vonthien $^{10} \cdot$ Kerstin Ratering $^{10}$. Christoph Schäfer ${ }^{11}$. Will Nelson Vance ${ }^{1}$ Paul Schmidt ${ }^{12}$

'Neuro-Urologisches Zentrum, Kliniken Beelitz GmbH, Beelitz Heilstätten, Deutschland

${ }^{2}$ Neurologisches Rehabilitationszentrum Quellenhof, Bad Wildbad, Deutschland

${ }^{3}$ Praxis für Neurologie Dr. Blersch, Regensburg, Deutschland

${ }^{4}$ Marianne-Strauß-Klinik, Behandlungszentrum Kempfenhausen für Multiple Sklerose Kranke gemeinnützige $\mathrm{GmbH}$, Berg, Deutschland

${ }^{5}$ Neurologisches Zentrum, Segeberger Kliniken, Bad Segeberg, Deutschland

${ }^{6}$ Querschnittgelähmten-Zentrum, Klinik für Paraplegiologie und Neuro-Urologie, Zentralklinik Bad Berka $\mathrm{GmbH}$, Bad Berka, Deutschland

${ }^{7}$ Sektion Neurourologie, Klinik für Urologie und Kinderurologie, Universitätsklinikum Bonn und NeuroUrologie, Neurologisches Rehabilitationszentrum der "Godeshöhe" e. V., Bonn, Deutschland

${ }^{8}$ Zentrum für Kontinenz und Neuro-Urologie, Kliniken Maria Hilf GmbH, Mönchengladbach, Deutschland

${ }^{9}$ Querschnittgelähmtenzentrum, BDH Klinik Greifswald gGmbH, Greifswald, Deutschland

${ }^{10}$ Coloplast GmbH, Hamburg, Deutschland

"Rehaklinik für Neurologie und Orthopädie, Johanniter-Klinik am Romberg, Dortmund, Deutschland

${ }^{12}$ Statistik, Berlin, Deutschland

\title{
Aktuelles zu neurogenen Dysfunktionen des unteren Harntraktes bei Multipler Sklerose
}

Die Diagnostik neurogener Funktionsstörungen des unteren Harntraktes (NLUTD) bei MS-Patienten ist eine besondere Herausforderung. In der Routine stellen sich folgende Fragen: 1. Wann sollten Patienten mit MS auf eine NLUTD hin untersucht werden? 2. Welche Patienten mit MS sollten gescreent werden? 3. Welche Screeningmethoden sind erforderlich? 4. Welche Screeningparameter geben Hinweise auf das Vorliegen einer NLUTD bei MS-Patienten? Eine multiprofessionelle Konsensusgruppe ging mit einer prospektiven Studie diesen Fragen nach und fand neue Antworten.

\section{Hintergrund und Fragestellung}

Bei Diagnosestellung der MS treten Symptome einer neurogenen Harnblasenfunktionsstörung eher selten auf [5],
10 Jahre danach liegt deren Prävalenz bereits bei $80 \%$ [27]. Manack et al. stellten bei der Analyse eines Kollektivs von 9315 MS-Patienten fest, dass das Bestehen einer NLUTD das Risiko für Krankenhauseinweisungen signifikant erhöht [21]. Die meisten Patienten berichten erst bei ihrer ersten urologischen Vorstellung, dass sie schon 10 Jahre unter Symptomen einer Blasenfunktionsstörung leiden [20]. Das bedeutet, dass den Patienten diese Lebensjahre verloren gehen, in denen sie eine deutlich eingeschränkte Lebensqualität infolge HWIs, Inkontinenz und Nierenkomplikationen in Kauf nehmen müssen, abgesehen von Verschlechterungen der MS durch diese Komplikationen [15, 18]. Mehr noch, über $56 \%$ der Patienten mit urologischen Symptomen erhalten keine Behandlung dieser Beschwerden, wie ein aktueller Report des deutschen MS-Registers aufzeigt [9].
Um die Situation zu verbessern, geben spezielle nationale Leitlinien Empfehlungen für eine strukturierte Diagnostik einer neurogenen Störung des unteren Harntraktes (NLUTD) bei MS. Leider liegen die Empfehlungen über eine sinnvolle Diagnostik teilweise weit auseinander (• Tab. 1).

Eine interprofessionelle Konsensusgruppe diskutierte diese Problematik und kam zu dem Schluss, die Relevanz der verschiedenen Parameter und der unterschiedlichen Expertenmeinungen im Rahmen einer multizentrischen Studie zu klären.

\section{Methoden}

Zwei Konsensuskonferenzen dienten der Auswertung der Literatur zu NLUTDs bei Patienten mit MS, der Sammlung relevanter Parameter und der Konzipierung einer prospektiven Evaluati- 


\section{Originalien}

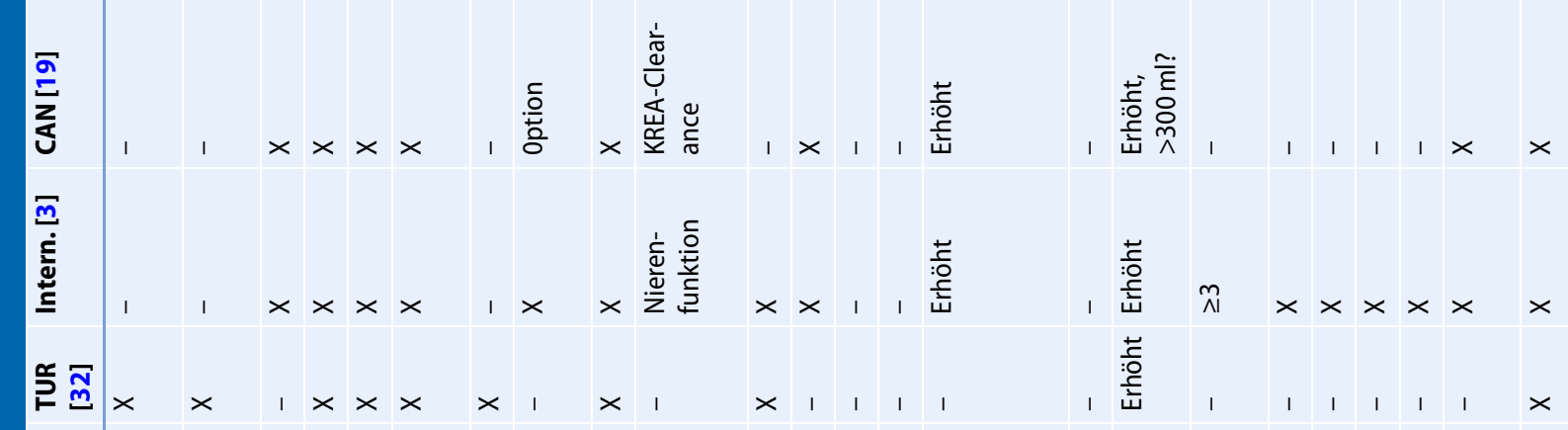

I

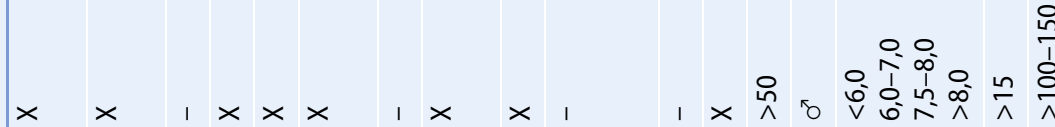

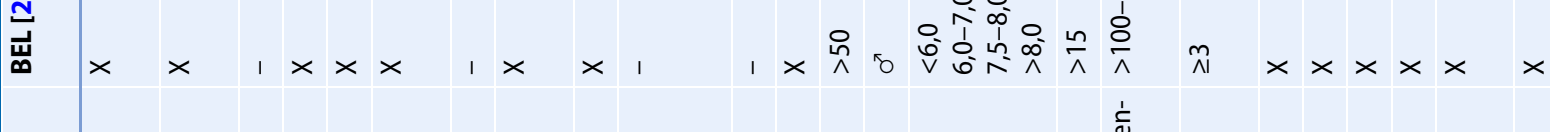

$\frac{E}{\Sigma}$

$\times$

$\times \quad 1 \times x$

$\times$ 崖 $\quad 1 \times$ 耑的 N

它

苂

$\times \times \quad \times \frac{\overleftrightarrow{\Psi}}{\underline{\Psi}}$

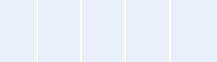

$\frac{0}{\stackrel{m}{m}}$

ฐ

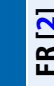

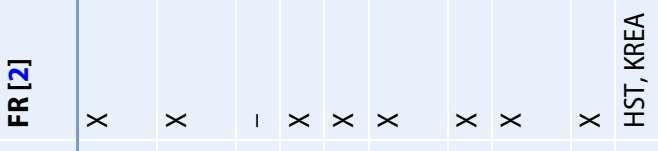

离

(1)

, $\frac{0}{N}, \quad, x \times x, \quad \times$

웅

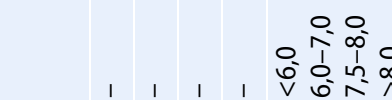

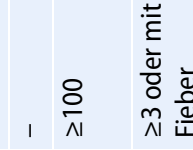

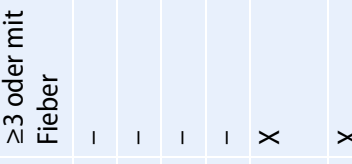

בับ

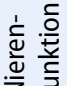

$\times \times 1, \stackrel{0}{n}$

, $\frac{\text { ก }}{N} N_{N}^{n} \times \times \times 1 \times$

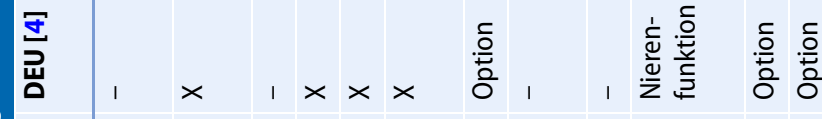

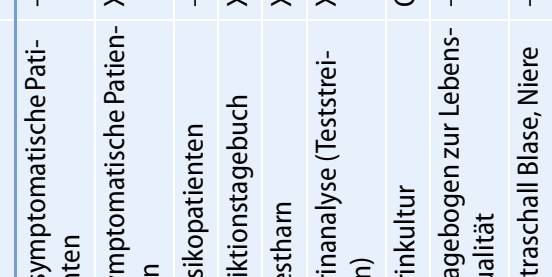

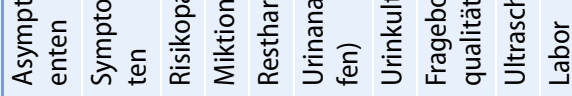

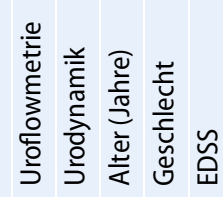

$\frac{8}{1}$

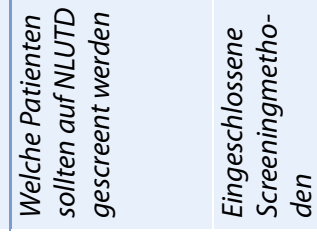

iू

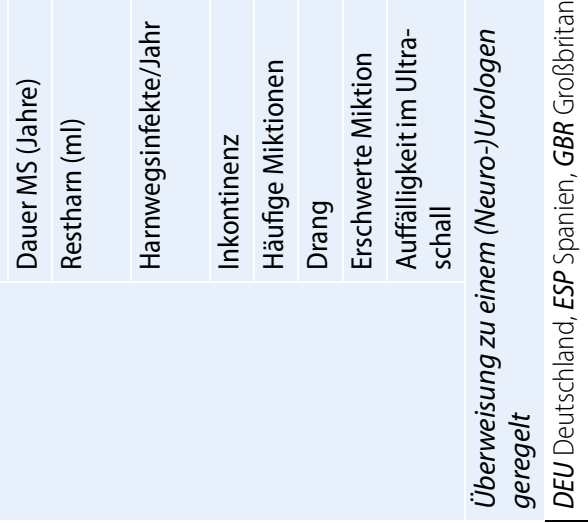


Hier steht eine Anzeige.

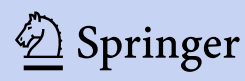


Nervenarzt 2021 · 92:349-358 https://doi.org/10.1007/s00115-020-01046-0

(c) Der/die Autor(en) 2021

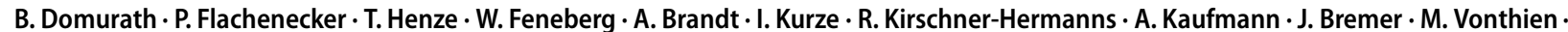 K. Ratering · C. Schäfer · W. N. Vance · P. Schmidt}

\section{Aktuelles zu neurogenen Dysfunktionen des unteren Harntraktes bei Multipler Sklerose}

\section{Zusammenfassung}

Hintergrund. In der Routine steht man vor der Aufgabe, neurogene Störungen des unteren Harntraktes (NLUTD) bei Patienten mit Multipler Sklerose (MS) frühzeitig zu erkennen und adäquat zu therapieren. Verschiedene nationale Leitlinien geben dazu sehr unterschiedliche praktische Empfehlungen.

Ziel der Arbeit. Erarbeitung eines einfachen, studienbasierten Algorithmus zum Nachweis von NLUTD bei Patienten mit MS, aus dem sich therapeutische Konsequenzen ableiten lassen. Material und Methode. Als direktes Ergebnis zweier multidisziplinärer Konferenzen wurde eine prospektive, multizentrische Studie initiiert. Deren Ziel war es, statistisch relevante Parameter für die Routinediagnostik von NLUTDs zu identifizieren. Als Goldstandard dienten Auffälligkeiten in der Urodynamik. In drei weiteren Konsensuskonferenzen wurden die Ergebnisse der Studie diskutiert, ein diagnostischer Algorithmus entwickelt und eine Erstlinientherapie konsentiert.

Ergebnisse und Diskussion. Der vorgeschlagene Algorithmus ermöglichte das Erkennen einer NLUTD bei Patienten mit MS mithilfe von 4 statistisch signifikanten Prädiktoren: (1) dem Restharnvolumen, (2) der Anzahl der Harnwegsinfektionen (HWI) innerhalb der letzten 6 Monate, (3) der standardisierten
Miktionsfrequenz und (4) dem Vorhandensein/Fehlen einer Harninkontinenz. Gestützt auf den Algorithmus benötigen ca. $75 \%$ der Patienten keine urodynamische Untersuchung zur First-line-Therapieentscheidung. In $25 \%$ der Fälle sind urodynamische Untersuchungen unerlässlich. Für die Routine notwendigen Assessments sind: die Anamneseerhebung, eine Restharnbestimmung, ein Miktionstagebuch und eine Uroflowmetrie (optional).

\section{Schlüsselwörter}

Multiple Sklerose · Neurogene Störungen des unteren Harntrakts (NLUTS) · Urodynamik .

Harnblasenfunktionsstörungen · Assessments

\section{Current aspects of neurogenic dysfunctions of the lower urinary tract in multiple sclerosis}

\section{Abstract}

Background. In the clinical management of patients with multiple sclerosis (MS), the challenge is to make an early diagnosis and initiate adequate treatment of neurogenic disorders of the lower urinary tract (NLUTD). Various national guidelines provide practical recommendations which are sometimes discordant.

Objective. To develop a simple evidencebased algorithm for detecting NLUTD in patients with MS that could be taken as a principle for deriving therapeutic consequences.

Material and methods. A prospective multicenter study was initiated as a direct result of two multidisciplinary conferences. The aim was to identify statistically and clinically relevant parameters for the routine diagnosis of NLUTD in patients with MS. Urodynamic abnormalities served as the gold standard. At three subsequent consensus conferences, the results of the study were discussed, a diagnostic algorithm was developed and consensus was reached on a first-line treatment.

Results and discussion. The proposed algorithm enables the detection of NLUTD in patients with MS with the help of four statistically significant predictors: 1 ) the residual urine volume, 2) the number of urinary tract infections (UTI) within the last 6 months, 3) the standardized micturition frequency and 4) the presence/absence of urinary incontinence. The newly developed algorithm has proved to be efficient with the following results: approximately $75 \%$ of the patients do not need a urodynamic examination for a first-line treatment decision. In $25 \%$ of cases, urodynamic examinations are essential for an adequate treatment decision. Routine assessments include the patient medical history, residual urine volume measurement, a micturition diary and a uroflowmetry (optional).

\section{Keywords}

Multiple sclerosis - Neurogenic lower urinary tract disorders (NLUTD) - Urodynamics . Bladder dysfunction · Assessments onsstudie. Nach der Studie wurden die Ergebnisse in zwei weiteren Konferenzen ausgewertet, ein diagnostischer Algorithmus etabliert und statistisch überprüft. Im Rahmen eines 5. Konsensustreffens konnten daraufhin Empfehlungen zum Basisscreening, zur Erstlinientherapie und zur erweiterten Diagnostik erarbeitet werden.

\section{Studiendesign}

Da urodynamisch nachweisbare NLUTDs bei MS-Patienten ohne urologische Sym- ptome keine Seltenheit sind, wurden diese Patienten in die Studie aufgenommen [28]. Die Einschlusskriterien waren: Alter über 18 Jahre und eine nach den McDonald-Kriterien diagnostizierte MS mit den typischen Verlaufsformen RRMS - schubförmig remittierend MS, SPMS - sekundär progrediente MS und PPMS - primär progrediente MS [29]. Ausgeschlossen wurden nur Patienten mit einem Dauerkatheter oder mit einer urodynamisch abgeklärten NLUTD.

Als Zielgröße diente eine abnormale Blasenfunktion in der Urodynamik (Bla- sendruckmessung). Alle Patienten waren aufgefordert, eine Uroflowmetrie (Harnstrahlmessung) durchzuführen. In jedem Fall erfolgte eine Restharnbestimmung. Alle Patienten füllten einen Anamnesefragebogen aus und führten ein Miktionsprotokoll über 3 Tage. Da die Miktionsfrequenz mit dem Ausscheidungsvolumen pro Tag im Zusammenhang steht, erfolgte eine Standardisierung der Miktionsfrequenz auf eine einheitliche Aus- 
Tab. 2 Patientencharakteristika des Studienkollektivs

\begin{tabular}{l|ll}
\hline Parameter & Mittelwert \pm Standardabweichung & Intervall \\
\hline $\begin{array}{l}\text { Alter (Jahre) } \\
\text { Geschlecht }\end{array}$ & $49,3 \pm 11,3$ & $19-75$ \\
\hline Männer: 33 & - \\
\hline EDSS & Frauen: 88 & $0,5-8,0$ \\
\hline MS-Dauer (Jahre) & $3,8 \pm 2,1$ & $0-42$ \\
MS-Verlauf & $12,2 \pm 9,6$ & - \\
& $\begin{array}{l}\text { RRMS }-56,8 \% \\
\text { SPMS }-31,3 \%\end{array}$ &
\end{tabular}

Tab. 3 Grenzwerte der Prädiktoren einer gestörten Funktion des unteren Harntraktes (NLUTD)

\begin{tabular}{lll} 
Parameter & Unauffällig & Auffällig \\
\hline Standardisierte Miktionsfrequenz & $\leq 13$ & $>13$ \\
\hline Kontinenz & Kontinenz & Inkontinenz \\
HWI-Rate/6 Monate & 0 & $>0$ \\
Restharn $(\mathrm{ml})$ & $<70$ & $\geq 70$
\end{tabular}

Tab. 4 Diagnostischer Algorithmus

\begin{tabular}{|c|c|c|c|c|c|}
\hline Methode & Prädiktoren & Gruppe 1 & Gruppe 2 & Gruppe 3 & Gruppe 4 \\
\hline $\begin{array}{l}\text { Miktionstage- } \\
\text { buch (3 Tage) }\end{array}$ & $\begin{array}{l}\text { Standardisierte } \\
\text { Miktionsfrequenz }\end{array}$ & $\leq 13$ & $>13$ & Variabel & $\leq 13$ \\
\hline Sonographie & Restharn (ml) & $<70$ & $<70$ & $\geq 70$ & $<70$ \\
\hline Anamnese & $\begin{array}{l}\text { HWI/6 Monate } \\
\text { und/oder Harnin- } \\
\text { kontinenz }\end{array}$ & Keine & Ja oder nein & Ja oder nein & Ja \\
\hline
\end{tabular}

scheidungsmenge von $2000 \mathrm{ml}$ nach folgender Formel:

Standardisierte Miktionsfrequenz $/ 24 \mathrm{~h}=$

Anzahl Miktionen in

48 Stunden $\times 2000 \mathrm{ml}$

Ausscheidungsmenge

in 48 Stunden $(\mathrm{ml})$

Für die Auswertung des Miktionsprotokolls wurden 2 aussagefähige Tage (48 h) ausgewählt.

\section{Statistik}

Um den Effekt verschiedener Parameter abzuschätzen, wurden logistische Regressionsmodelle herangezogen. Die Auswahl der relevanten Parameter erfolgte mithilfe einer schrittweisen Selektion der Variablen nach dem AkaikeInformationskriterium (AIC; [1]). Die Schwellenwerte der Prädiktoren wurden unter Verwendung einer rekursiven Partitionierung identifiziert [6]. Schlussendlich erfolgte die Einschätzung der prognostischen Qualität ausgewählter Klassifikatoren für die Vorhersage ei-
- Rate an HWIs in den vorausgegangenen 6 Monaten $(\mathrm{OR}=2,03$; $\mathrm{KI}$ : [1,04-6,68]),

- Inkontinenz (OR=3,93; KI:[1,17$15,7])$ und

- Restharn (OR=1,25; KI:[1,07-1,62]).

Für die 4 oben genannten Prädiktoren wurden statistische Grenzwerte ermittelt, bei denen sich urodynamische Auffälligkeiten mit hoher Sicherheit erwarten ließen (•Tab. 3).

Die 4. Konsensuskonferenz entwickelte einen 3-mal-4-Felder-Algorithmus. Die 3 Methodenzeilen wurden aus den Prädiktoren gebildet (• Tab.4).

Wenn man die Grenzwerte der 4 Prädiktoren kombiniert, ergeben sich 16 mögliche Kombinationen (Cluster). Nach statistischer Überprüfung verblieben davon 4 Cluster, aus denen sich die 4 Gruppenspalten des Algorithmus ergaben (•Tab. 4). Die Sensitivität bezüglich einer urodynamischen Auffälligkeit lag in diesem 3-mal-4-Algorithmus bei $95 \%$, der positive Voraussagewert bei $91 \%$.

Insgesamt zeigte sich ein geringer Anteil an Patienten mit einer unauffälligen Urodynamik (14\%) in unserem Studienkollektiv.

Der Gruppe 1 wurden Patienten zugeordnet, die die Grenzwerte der Prädiktoren nicht erreichten (keine urologischen Symptome). Zu erwarten wäre, dass sich in dieser Gruppe mit unauffälliger Symptomatik keine oder nur sehr wenige Fälle mit urodynamischen Auffälligkeiten finden lassen. In dieser Gruppe hatten aber nur 11 der 23 Patienten einen unauffälligen, 12 Patienten einen auffälligen urodynamischen Befund.

Patienten der Gruppe $2 \quad(n=23$, $19,0 \%)$ fielen vor allem durch eine erhöhte standardisierte Miktionsfrequenz auf, bei unauffälligem Restharn. Diese Patienten hatten alle urodynamische Auffälligkeiten.

Das Hauptmerkmal der größten Gruppe von Patienten in unserem Studienkollektiv (Gruppe 3, $n=38,31,4 \%$ ) war eine Restharnmenge von $\geq 70 \mathrm{ml}$. Alle Patienten mit einer normalen Urodynamik hatten einen Restharn unter $70 \mathrm{ml}$. In der Gruppe 3 fanden sich so- 


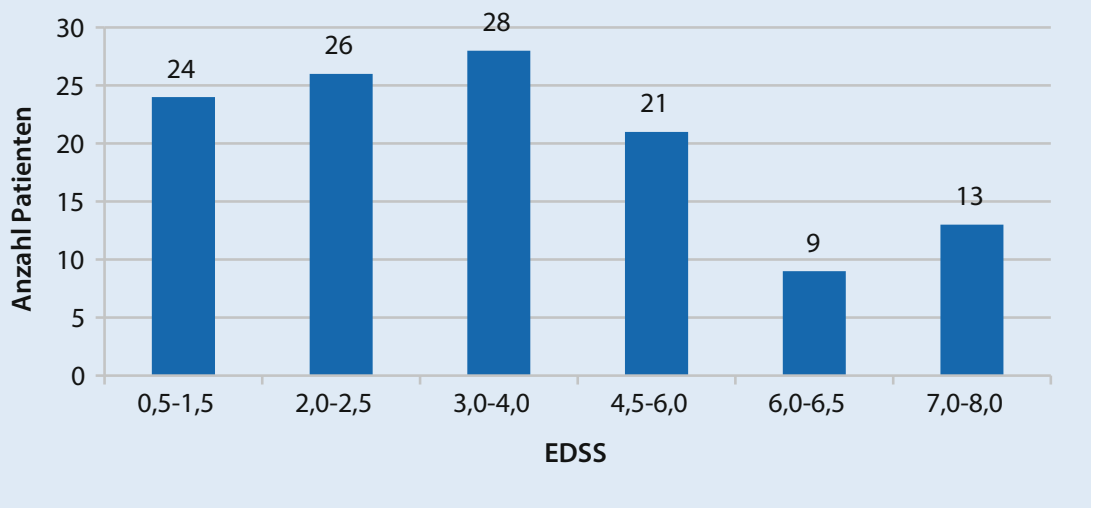

Abb. $1 \Delta$ Verteilung des EDSSin der Studienpopulation $(n=121)$

mit nur Patienten mit einer auffälligen Urodynamik.

Patienten der Gruppe $4 \quad(n=33$, $27,2 \%)$ unterscheiden sich von denen der Gruppe 1 durch das Vorliegen einer Harninkontinenz und/oder von vorausgegangenen HWIs. Diese Gruppe ist urodynamisch sehr heterogen $(15,2 \%$ - normale Urodynamik, $20 \%$ - Inkontinenz unterschiedlichen Typs, $27 \%$ - Detrusorunteraktivität, 37,5\% - Detrusorüberaktivität, $20 \%$ - veränderter Harndrang und $40 \%$ - Detrusor-Sphinkter-Dyssynergie).

\section{Diskussion}

Die vorliegende Studie bestätigt, dass der Anteil urodynamisch feststellbarer NLUTDs bei Patienten mit MS hoch ist. In unserer Studie hatten $86,0 \%$ aller Patienten eine auffällige Urodynamik. Dieser hohe Prozentsatz ist vergleichbar mit dem bei Ineichen et al. - 86,5\% und bei Wiedemann et al. - 78,0\%, auch wenn man berücksichtigt, dass sich die Einschlusskriterien dieser Studien von denen unserer Studie unterscheiden [17, 31]. Die von uns ursprünglich ins Auge gefasste risikoadaptierte Gruppenbildung im primär entworfenen Algorithmus (niedriges, mittleres, hohes Risiko für eine abnormale Urodynamik) musste deshalb aufgegeben werden. Sogar Patienten ohne urologische Symptome hatten in 52,2\% der Fälle auffällige urodynamische Befunde, sodass der Begriff „niedriges Risiko“ nicht zutraf. Der Anteil auffälliger Urodynamiken in den Gruppen mit einem angenommenen mittleren und hohen Risiko war so hoch und lag in beiden Gruppen so nahe beieinander $(84,6 \%$ und $92,6 \%)$, dass sich deren Prognosegüte nicht mehr voneinander unterschied. Als weiteres Problem wurden die aus der Literatur entnommenen "red flags“ identifiziert, die nicht validiert waren (• Tab. 1). Deshalb erfolgte eine Umorientierung auf die statistische Bestätigung von Parametern als Prädiktoren und auf deren statistisch ermittelte Grenzwerte. Das erlaubte eine Umstellung auf einen therapeutisch orientierten Algorithmus nach statistisch validierten Diagnosekriterien.

Entgegen der Ausführungen mancher Leitlinien (• Tab. 1) konnten wir die Dauer der MS, das Alter, das Geschlecht und den MS-Verlauf nicht als Prädiktoren einer NLUTD identifizieren. Wir bestätigen damit die Ergebnisse anderer Studien. Auch der EDSS war für die Vorhersage einer NLUTD ungeeignet $(\mathrm{OR}=0,91$; $\mathrm{KI}:[0,69-1,2])$. Ineichen et al. fanden bei Patienten mit MS einen EDSS-Cutoff von 5,0 für das Risiko einer Schädigung des oberen Harntraktes [17]. Dieses theoretische Risiko für Patienten mit MS definierten Ineichen et al. nach Kriterien, die ursprünglich für querschnittgelähmte Patienten ermittelt worden waren. Sie ermittelten in $87 \%$ ein theoretisches Risiko für eine Schädigung des oberen Harntraktes bei MS-Patienten. Eine tatsächliche Nierenfunktionsschädigung ist aber bei Patienten mit MS im Gegensatz zu querschnittgelähmten Patienten ein Ereignis im eher niedrigen einstelligen Prozentbereich [26, 27]. Giannantoni et al. stellten eine Korrelation zwischen dem EDSS und einer Detrusorunteraktivität fest, wobei nur 15,4\% der Patienten mit einer auffälligen Urodynamik eine Unteraktivität aufwiesen [13]. Wiedemann et al. fanden einen EDSS von $>6,5$ (Rollstuhlabhängigkeit) als Prädiktor einer NLUTD [31]. Dieser Prädiktor aber ist sehr schwach, da er nur $14,0 \%$ der Patienten mit einer auffälligen Urodynamik identifizierte. In unserer Studie wären es nur $11,4 \%$. Wir halten deshalb eine EDSS-basierte Risikostratifizierung, wie in einigen Leitlinien vorgeschlagen (• Tab. 1), für wenig Erfolg versprechend.

Im Jahr 2012 bestätigten Tadayyon et al. frühere Untersuchungen über einen hohen Anteil an urodynamischen Auffälligkeiten bei MS-Patienten, die keine urologischen Beschwerden angaben (62\%; [13]). In unserer Studie waren es in der Gruppe $152,1 \%$. Tadayyon et al. fanden im Verlauf, dass $94 \%$ dieser Patienten innerhalb eines Jahres urologische Symptome entwickelten. Einige nationale Leitlinien empfehlen ein Basisscreening für Patienten, die keine urologischen Symptome angeben (- Tab. 1). Unsere Ergebnisse untermauern diese Empfehlung. Um Patienten mit einer verdeckten NLUTD herauszufinden, kann eine Uroflowmetrie statt einer Urodynamik eingesetzt werden. Wir bestimmten die OR der Uroflowmetrie bezüglich einer auffälligen Urodynamik in unserer Studie mit 4,80 (KI: [1,4-19,21]), die Sensitivität mit 79,3\% und die PPV mit 94,0\%. Symptomlose Patienten mit auffälligem Uroflow benötigen nach unserer Meinung keine Therapie, sie sollten aber in kürzeren Abständen kontrolliert werden (Konsensusempfehlung: nach 6 Monaten, - Tab. 5). Beim Rescreening geht es darum, ob die Grenzwerte der Prädiktoren überschritten werden.

Kennzeichnend für die 2. Gruppe ist eine erhöhte Miktionsfrequenz $(\geq 13$ $\mathrm{mal} / 24 \mathrm{~h}$ ), deren Standardisierung für die Anwendung im Algorithmus entscheidend ist. Die nichtstandardisierte Miktionsfrequenz schwankte von $4-\mathrm{mal} / 24$ bis 23-mal/24h bei Ausscheidungsmengen zwischen 600 und $3000 \mathrm{ml}$. Nach Haggiag et al. taugt die nichtstandardisierte Miktionsfrequenz mit einer Sensitivität von nur 26,3\% nicht als Prädiktor einer gestörten Harnblasenfunktion [14]. 
Hier steht eine Anzeige.

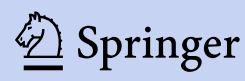




\begin{tabular}{lllll} 
& Gruppe 1 & Gruppe 2 & Gruppe 3 & Gruppe 4 \\
\hline Erweiterte Diagnostik im Rahmen des Basisscreenings & Uroflowmetrie & Keine & Keine & Urodynamik! \\
Erstlinientherapie & Keine & $\begin{array}{l}\text { Antimuskarinika } \\
\text { (cave: Restharn) }\end{array}$ & ISK, evtl. Antimuskarinika & Keine
\end{tabular}

Die standardisierte Miktionsfrequenz korrelierte in unserer Studie gut mit einer Detrusorüberaktivität $(\mathrm{OR}=1,16$; $\mathrm{KI}:[1,07-2,06])$, sodass „first-line“ eine orale oder transdermale detrusordämpfende Therapie mit Antimuskarinika gerechtfertigt ist (- Tab. 5; [16]).

In allen Leitlinien wird bei einem erhöhten Restharn der intermittierende Selbstkatheterismus (ISK) empfohlen, wobei uneinheitliche Grenzwerte eines pathologischen Restharns festgelegt wurden (• Tab. 1). Unser Grenzwert von $70 \mathrm{ml}$ Restharn in der Gruppe 3 ist relativ niedrig, ähnelt aber dem in der kleinen Studie von Tracy et al. [30]. Der Cutoff bei $70 \mathrm{ml}$ dient primär dem Aufdecken einer NLUTD bei MS-Patienten. Die Zweckmäßigkeit und Durchführbarkeit des ISK muss im Einzelfall geprüft werden und kann entsprechend der 5. Konsensuskonferenz nicht allein an einer konkreten Höhe des Restharns festgemacht werden (Gruppe 3, - Tab. 5). Ein ISK setzt ausreichende kognitive, visuelle und sensomotorische Fähigkeiten voraus, die bei MS-Patienten nicht immer gegeben sind. In der 3. Gruppe korrelierte die Restharnmenge nicht mit der HWI-Rate, wie bereits aus anderen Studien bekannt [7]. Eine Patientin mit einem Restharn von $100 \mathrm{ml}$ hatte 8 HWIs in den letzten 6 Monaten. In der Gruppe mit Restharnmengen zwischen 200 und $300 \mathrm{ml}$ hatte keiner der Patienten einen HWI $(n=9)$. Für eine individuelle Handhabung des Katheterismus spricht auch die Tatsache, dass der Anteil von Patienten mit Detrusorüberaktivität in der 3. Gruppe relativ hoch ist (59,5\%), bei einem Miktionsfrequenzmedian von 16-mal/24h und einem Restharnmedian von $172 \mathrm{ml}$. Bei der Therapieentscheidung für ein den Detrusor dämpfendes Medikament ist mit der Zunahme der Restharnmenge zu rechnen. Ein Urologe sollte frühzeitig einbezogen werden.

Die Resultate unserer Studie ergaben, dass Patienten der Gruppe $4 \quad(n=33$,
$27,3 \%$ ) sehr unterschiedliche urodynamische Befunde bieten. Deshalb fehlt bei diesen Patienten ein First-line-Therapieansatz. Vor einer Therapieentscheidung halten wir deshalb eine urodynamische Abklärung bei jedem Patienten dieser Gruppe für sinnvoll (• Tab. 5). Zum Beispiel waren 67,5\% der Patienten der 4. Gruppe inkontinent, von diesen hatten aber nur $26 \%$ eine Detrusorüberaktivität. Das verdeutlicht, dass eine antimuskarine Therapie zur Behebung der Inkontinenz bei Patienten der Gruppe 4 wenig Aussicht auf Erfolg hat. Die aktuellen Leitlinien des National Institutes for Health and Care Excellence (NICE) empfehlen, bei Patienten mit MS keine urodynamischen Untersuchungen zu veranlassen [23]. Dieser Argumentation kann aus unserer Sicht nicht gefolgt werden. Die meisten Leitlinien sehen das ebenso (•Tab. 1).

Zusammenfassend zeigen die Ergebnisse dieser Studie, dass eine NLUTD bei Patienten mit MS mit relativ geringem Aufwand sicher diagnostiziert werden kann, ohne dass dazu eine invasive Urodynamik erforderlich ist. Eine Firstline-Therapie kann nach Zuordnung zu den einzelnen Gruppen aus dem vorgeschlagenen Algorithmus abgeleitet werden. Es empfiehlt sich aber, eine enge Beziehung zu einem niedergelassenen Urologen aufzubauen, der wünschenswert eine Uroflowmetrie vorhält. Die meisten Querschnittzentren und einige spezialisierte Rehabilitationszentren verfügen über eine qualifizierte Neurourologie. Sie sind zwar im Allgemeinen nicht wohnortnah gelegen, angesichts deren Expertise bei neurourologischen Fragestellungen sollte man aber den Kontakt zu diesen Zentren auch bei MS suchen [8].

\section{Limitationen}

- Eine urodynamisch nachweisbare NULTD war bei den Patienten in unserer Studie häufig nachzuweisen.
Um die Aussagekraft des Algorithmus zu verfeinern, sind mehr Patienten mit unauffälliger Urodynamik erforderlich.

- Die Gruppe der Patienten, die keine urologischen Symptome angab, war relativ klein, sodass wegen der praktischen Bedeutung eine Bestätigung unserer Ergebnisse in einer größeren Studie wünschenswert wäre.

- Unsere Studie bestätigt die diagnostische Relevanz eines Restharns ab $70 \mathrm{ml}$ bei Patienten mit MS. Die Bestimmung eines therapeutisch relevanten Restharngrenzwertes im Kontext mit anderen klinischen Parametern müsste in zukünftigen Studien geklärt werden.

- Die aus dem Algorithmus abgeleiteten Therapiemaßnahmen sind im Expertenkonsensus entstanden und bislang nicht auf ihren Erfolg hin in der Praxis evaluiert worden.

\section{Fazit für die Praxis}

- Vor jedem Screening sollte ein akuter HWI ausgeschlossen werden, da die Symptome eines HWI den Symptomen einer NLUTD bei MS-Patienten sehr ähnlich sind.

- Ein Patientenfragebogen und ein Miktionsprotokoll bilden das diagnostische Grundgerüst, um NLUTDs bei Patienten mit MS frühzeitig erkennen zu können.

- Eine Restharnbestimmung ist unverzichtbar.

- Die Schwellenwerte für die standardisierte Miktionsfrequenz ( $>13$ $\mathrm{mal} / 24 \mathrm{~h}$ ), für den Restharn ( $\geq 70 \mathrm{ml}$ ) sowie für die Rate von HWls ( $>0$ mal/6 Monate) und einer Harninkontinenz ( $j a / n e i n)$ erlauben eine Einordnung in einen vorgeschlagenen Algorithmus und eine Erstlinientherapie. 
- Patienten ohne festzustellende urologische Auffälligkeiten weisen in über $50 \%$ eine neurogene Dysfunktion des unteren Harntraktes auf und sollten einer Uroflowmetrie zugeführt werden, um eine verdeckte NLUTD nichtinvasiv erkennen zu können.

\section{- Eine urodynamische Untersuchung} muss für bestimmte Patienten vor einer sinnvollen Therapieentscheidung im Behandlungskonzept berücksichtigt werden.

\section{Korrespondenzadresse}

Dr. Burkhard Domurath
Neuro-Urologisches Zentrum,
Kliniken Beelitz GmbH
Paracelsusring 6A,
14547 Beelitz Heilstätten,
Deutschland
bdomurath@yahoo.de

\section{Einhaltung ethischer Richtlinien}

Interessenkonflikt. B. Domurath erhielt Vortragshonorare und Beratungskosten von Wellspect, Allergan und Coloplast. Interessenkonflikte bestehen nicht. P. Flachenecker erhielt Honorare für Vorträge und Beratungstätigkeiten von den Firmen Almirall, Bayer, Biogen Idec, Genzyme, Novartis, Merck Serono, Roche und Teva und hat an industriegesponsorten Studien von Biogen Idec und Novartis teilgenommen. Interessenkonflikte bestehen nicht. T. Henze erhielt Vortragshonorare und Aufwandsentschädigungen für Advisory Boards von Almirall, Coloplast, Genzyme, Novartis, Sanofi. Interessenkonflikte bestehen nicht. W. Feneberg erhielt Vortragshonorare, Aufwandsentschädigungen für Advisory Boards bzw. Unterstützung bei Fortbildungen von Almirall, Bayer, Biogen Idec Coloplast, Genzyme, Ipsen, Merz, Novartis, SanofiAventis. Interessenkonflikte bestehen nicht. A. Brandt erhielt Vortragshonorare, Aufwandsentschädigungen für Advisory Bords bzw. Unterstützung bei Fortbildungen von Bayer, Biogen Idec, Coloplast, Genzyme, Merk Serono, Novartis, Roche, Sanofi-Aventis, Teva. Ein Interessenkonflikt besteht nicht. I. Kurze erhielt Honorare für Vorträge, Beratungstätigkeit und Publikationen von Coloplast, Georg Thieme Verlag, Oxyton, Teleflex, Wellspect, Medtronic. Ein Interessenkonflikt besteht nicht. R. Kirschner-Hermanns ist Gründer und Eigentümer der aus der BMBF Forschungintiative "Industrie in Klinik" hervorgegangenen Firma MEC-ABC GmbH und Vorsitzende des Fördervereins Kontinenzforschung und Kontinenzaufklärung e. V. Ein Interessenkonflikt besteht nicht. A. Kaufmann erhielt Vortragshonorare, Aufwandsentschädigungen für Advisory Boards, bzw. Unterstützung bei Fortbildungen von Apogepha, Coloplast, Sanofi, Oxyton und Farco-Pharm. Ein Interessenkonflikt besteht nicht. J. Bremer erhielt Vortragshonorare und Beratungskosten von Apogepha, Allergan, und Coloplast. Ein Interessenkonflikt besteht nicht. M. Vonthien war als Mitarbeiterin von Coloplast an der Initiierung und den organisatorischen Vorbereitungen dieses Vorhabens beteiligt sowie an den
Literaturrecherchen. Sie designte die Fragebögen und Miktionsprotokolle der Konsensusgruppe und beteiligte sich am Design des Algorithmus. Weder auf die Auswertung der Resultate noch auf die Diskussionen und den Inhalt der Publikation nahm sie Einfluss. K. Ratering war als Mitarbeiterin von Coloplast an der Literaturbeschaffung zum aktuellen Stand der neurologischen und urologischen Diagnostik und Therapie beteiligt sowie am Design des Algorithmus. Sie beteiligte sich nicht an der Auswertung, Besprechung und Diskussion der Resultate. P. Schmidt erhielt für die statistische Auswertung der Studie ein Honorar von Coloplast. C. Schäfer und W.N. Vance geben an, dass kein Interessenkonflikt besteht.

Für diesen Beitrag wurden von den Autoren keine Studien an Menschen oder Tieren durchgeführt. Für die aufgeführten Studien gelten die jeweils dort angegebenen ethischen Richtlinien.

Open Access. Dieser Artikel wird unter der Creative Commons Namensnennung 4.0 International Lizenz veröffentlicht, welche die Nutzung, Vervielfältigung, Bearbeitung, Verbreitung und Wiedergabe in jeglichem Medium und Format erlaubt, sofern Sie den/die ursprünglichen Autor(en) und die Quelle ordnungsgemäß nennen, einen Link zur Creative Commons Lizenz beifügen und angeben, ob Änderungen vorgenommen wurden.

Die in diesem Artikel enthaltenen Bilder und sonstiges Drittmaterial unterliegen ebenfalls der genannten Creative Commons Lizenz, sofern sich aus der Abbildungslegende nichts anderes ergibt. Sofern das betreffende Material nicht unter der genannten Creative Commons Lizenz steht und die betreffende Handlung nicht nach gesetzlichen Vorschriften erlaubt ist, ist für die oben aufgeführten Weiterverwendungen des Materials die Einwilligung des jeweiligen Rechteinhabers einzuholen.

Weitere Details zur Lizenz entnehmen Sie bitte der Lizenzinformation auf http://creativecommons.org/ licenses/by/4.0/deed.de.

\section{Literatur}

1. Akaike H (1973) Information theory and an extension of the maximum likelihood principle. In: Petrov BN, Csáki F (Hrsg) 2nd International symposium on information theory. Akadèmiai Kiadò, Budapest, S267-281

2. Amarenco $G$, Chartier-Kastler $E$, Denys $P$ et al (2013) First-line urological evaluation in multiple sclerosis: validation of a specific decision-making algorithm. Mult Scler 19:1931-1937

3. Averbeck MA, lacovelli V, Panicker J et al (2020) Urodynamics in patients with multiple sclerosis: a consensus statement from a urodynamic experts working group. Neurourol Urodyn 39:73-82

4. AWMF (2020) Diagnose und Therapie der Multiplen Sklerose, Neuromyelitis Optica Spektrum und MOG-IgG-assoziierte Erkrankungen. https://www. awmf.org/leitlinien/detail/anmeldung/1/I/030050.html.Zugegriffen:25. Sept. 2020

5. Bakke A, Myhr KM, Grønning Metal (1996) Bladder, bowel and sexual dysfunction in patients with multiple sclerosis-A cohort study. Scand J Urol Nephrol Suppl 179:61-66

6. Breiman L, Friedman JH, Olshen RA et al (2017) Classification and regression trees. CRC, Boca Raton https://doi.org/10.1201/9781315139470
7. Brookman-May S, Burger M, Hoschke B et al (2010) Assoziation zwischen Restharnvolumen und Harnwegsinfektion: Prospektive Studie an 225 Männern. Urologe A 49:1163-1168

8. Deutschsprachige Medizinische Gesellschaft für Paraplegie (DMGP) (2014) Manual zur neurourologischen Diagnostik und Therapie Querschnittgelähmter.https://www.dmgp.de/images/ stories/AK-Neuro-Urologie-Manual-2014.pdf. Zugegriffen: 15. Juni 2020(S. 95-98)

9. Flachenecker P, Eichstädt K, Berger K et al (2020) Multiple Sklerose in Deutschland: aktualisierte Auswertungen des MS Registers der DMSG 2014-2018. Fortschr Neurol Psychiatr 88:436-450

10. Fowler C, Panicker J, Drake M et al (2009) A UK consensus on the management of the bladder in multiple sclerosis. J Neurol Neurosurg Psychiatry 80:470-477

11. Ghezzi A, Carone R, Del Popolo G et al (2011) Recommendations for the management of urinary disorders in multiple sclerosis: a consensus of the Italian multiple sclerosis study group. Neurol Sci 32:1223-1231

12. Ghezzi A, Muuta E, Bianchi F et al (2016) Diagnostic tools for assessment of urinary dysfunction in MS patients without urinary disturbances. Neurol Sci 37:437-442

13. Giannantoni A, Scivoletto G, Di Stasi SM et al (1999) Lower urinary tract dysfunction and disability status in patients with multiple sclerosis. Arch Phys Med Rehabil 80:437-441

14. Haggiag S, Bolla G, Picconi O et al (2017) Discrepancies between urinary symptoms assessment and objective bladder dysfunctions in multiple sclerosis. Mult Scler Demyelinating Disord 2:11

15. Hemmett L, Holmes J, al Barneset M (2004) What drives quality of life in multiple sclerosis? QJM 97:671-676

16. Henze $T$, Feneberg $W$, Flachenecker $P$ et al (2018) Neues zur symptomatischen Therapie bei MS-Therapie: Teil 3 Blasenfunktionsstörungen. Nervenarzt 89:184-192

17. Ineichen BV, Schneider MP, Hlavica M et al (2018) High EDSS can predict risk for upper urinary tract damage in patients with multiple sclerosis. Mult Scler 24:529-534

18. Jick SS, Li L, Falcone GJ, Vassilev ZP et al (2015) Epidemiology of multiple sclerosis: results from a large observational study in the UK. J Neurol 262:2033-2041

19. Kavanagh A, Baverstock R, Campeau L et al (2019) Urological association guideline: diagnosis, management, and surveillance of neurogenic lower urinary tract dysfunction-Executive summary. Can Urol Assoc J 13:156-165

20. Koldewijn EL, Hommes OR, Lemmens WA et al (1995) Relationship between lower urinary tract abnormalities and disease-related parameters in multiple sclerosis. JUrol 154:169-173

21. Manack A, Motsko SP, Haag-Molkenteller C et al (2011) Epidemiology and healthcare utilization of neurogenic bladder patients in a US claims database. Neurourol Urodyn 30:395-401

22. Medina-Polo J, Adot JM, Allué $M$ et al (2020) Consensus document on the multidisciplinary management of neurogenic lower urinary tract dysfunction in patients with multiple sclerosis. Neurourol Urodyn 39:762-770

23. NICE (2012) Urinary incontinence in neurological disease: assessment and management. https://www.nice.org.uk/guidance/cg148/ resources/urinary-incontinence-in-neurologicaldisease-assessment-and-management-pdf- 


\section{Originalien}

35109577553605. Zugegriffen: 25. Sept. 2020 (S.17)

24. De Ridder D, Van der Aa F, Debruyne J et al (2013) Consensus guidelines on the neurologist's role in the management of neurogenic lower urinary tract dysfunction in multiple sclerosis. Clin Neurol Neurosurg 115:2033-2040

25. Seland TP, Brunette J, Clesson IMetal (1999) Urinary dysfunction in multiple sclerosis. Multiple sclerosis council for clinical practice guidelines. https:// pva-cdnendpoint.azureedge.net/prod/libraries/ media/pva/library/publications/urinary_ms79c8. pdf.Zugegriffen:5. Okt. 2019

26. Shakir NA, Satyanarayan A, Eastman J et al (2019) Assessment of renal deterioration and associated risk factors in patients with multiple sclerosis. Urology 123:76-80

27. De Sèze $M$, Ruffion $A$, Denys $P$ et al (2007) The neurogenic bladder in multiple sclerosis: review of the literature and proposal of management guidelines. Mult Scler 13:915-928

28. Tadayyon F, Etemadifar M, Bzeih H et al (2012) Association of urodynamic findings in new onset multiple sclerosis with subsequent occurrence of urinary symptoms and acute episode of disease in females.J Res Med Sci 17:382-385

29. Thompson AJ, Banwell BL, Barkhof F et al (2018) Diagnosis of multiple sclerosis: 2017 revisions of the McDonald criteria. Lancet Neurol 17:162-173

30. Tracy TF, Riser ES, Bladder MB (2014) Dysfunction and disability in people newly diagnosed with multiple sclerosis. Int J MS Care 16:105 (Annual Meeting Consortium of Multiple Sclerosis Centers, abstract SX22)

31. Wiedemann A, Kaeder M, Greulich W et al (2013) Which clinical risk factors determine a pathological urodynamic evaluation in patients with multiple sclerosis? An analysis of 100 prospective cases. World JUrol 31:229-233

32. Çetinel B, Tarcan T, Demirkesen 0 et al (2013) Management of lower urinary tract dysfunction in multiple sclerosis: a systematic review and Turkish consensus report. Neurourol Urodyn 32:1047-1057

33. Çinar BP, Yorgun YG (2018) What we learned from the history of multiple sclerosis measurement: expanded disability status scale. Noro Psikiyatr Ars 55:S69-S75

\section{Hier steht eine Anzeige.}

\section{Springer}

